\title{
TOR signaling in invertebrates
}

\author{
Alexandre Soulard ${ }^{1}$, Adiel Cohen ${ }^{1}$ and Michael $\mathrm{N}$ Hall
}

\begin{abstract}
The Target of Rapamycin (TOR), a protein kinase, is the central node of a highly conserved signaling network that regulates cell growth in response to nutrients, hormones, and stresses. TOR is found in two functionally distinct complexes, TORC1 and TORC2. In this review we address the most recent advances in TOR signaling in invertebrate model organisms, including yeasts, plants, worms, and insects.
\end{abstract}

\section{Address}

Biozentrum, University of Basel, Basel $\mathrm{CH}-4056$, Switzerland

Corresponding author: Hall, Michael N (m.hall@unibas.ch)

${ }^{1}$ These authors contribute equally to this review.

\author{
Current Opinion in Cell Biology 2009, 21:825-836 \\ This review comes from a themed issue on \\ Cell division, growth and death \\ Edited by Angelika Amon and Mike Tyers \\ Available online 19th September 2009 \\ 0955-0674/\$ - see front matter \\ (C) 2009 Elsevier Ltd. All rights reserved. \\ DOI 10.1016/j.ceb.2009.08.007
}

\section{Introduction}

Invertebrate model organisms are valuable in biomedical research. Rapamycin was identified in the early 1970s as an antifungal agent against the pathogenic yeast Candida albicans $[1,2]$ but was later developed as an immunosuppressive for use in the prevention of allograft rejection. In the early 1990s, the Target of Rapamycin (TOR) was discovered in Saccharomyces cerevisiae [3]. Soon thereafter, TOR was identified in many other organisms ranging from yeasts to mammals. More recently, TOR was shown to play a prominent role in many human disorders in addition to allograft rejection, including cancer, diabetes, and cardiovascular disease. TOR is a member of the PIKK family, a family of atypical serine/threonine kinases that also includes DNA-PK, ATM, and TEL1. It is found in two conserved complexes termed TOR complex 1 (TORC1) and TOR complex 2 (TORC2) [4]. TORC1 and TORC2 are essential regulators of cell growth in response to nutrients, hormones, or stresses. TORC1 mediates temporal control of cell growth by activating anabolic processes such as ribosome biogenesis, protein synthesis, transcription, and nutrient uptake and by inhibiting catabolic processes such as autophagy and ubiquitin-dependent proteolysis [4]. In contrast, TORC2 mediates spatial control of cell growth mainly by regulating actin cytoskeleton organization. Invertebrates also played a major role in elucidating these generally conserved features of the TOR signaling network. Here we review the major findings of the last two years on TOR signaling and cell growth regulation in invertebrates.

\section{TOR complexes: composition, structure, and localization}

The TOR complexes were originally described in $S$. cerevisiae, but have now been identified in a wide variety of organisms ranging from yeast to mammals (see Table 1). In S. cerevisiae, the core components of TORC1 are TOR (TOR1 or TOR2), KOG1, and LST8. The core components of budding yeast TORC2 are TOR2, AVO1, AVO3, and LST8. The corresponding components in other species are listed in Table 1 . In addition to the core components, both complexes contain speciesspecific subunits (Table 1) [4]. During the last two years, TORC1 and TORC2 complexes have been identified biochemically or genetically in the fission yeast Schizosacchromyces pombe $\left[5,6^{\bullet \bullet}\right]$, in the pathogenic yeast $C$. albicans [7], in the unicellular green alga Chlamydomonas reinhardtii [8] and in the protozoan Trypanosoma brucei $\left[9^{\bullet}\right.$, which emphasizes the high degree of conservation of these complexes in eukaryotes. As in S. cerevisiae, fission yeast contains two TOR genes, TOR1 and TOR2. However, because of the naming of the fission yeast TORs based on order of discovery rather than function, $S$. pombe SpTOR1 corresponds to budding yeast TOR2 and vice versa. SpTOR2 is found mainly in TORC1, associated with WAT1/LST8, MIP1/KOG1, TCO89, and the uncharacterized protein TOC1 $\left[5,6^{\bullet \bullet}\right.$, whereas SpTOR1 is found mainly in TORC2 associated with WAT1/LST8, SIN1/AVO1, STE20/AVO3, and BIT61 [5,6 ${ }^{\bullet \bullet}$. Interestingly, all the so far identified, conserved TORC subunits are highly phosphorylated. Furthermore, both SpTOR1 and Sp'TOR2 associate with the casein kinase II ORB5, the PIKK regulator TEL2 and the uncharacterized protein TTI1 $\left[6^{\bullet \bullet}\right.$. TEL2 appears to interact with all PIKKs and, at least in mammals, it maintains the stability of the TOR protein, thereby indirectly influencing TOR downstream signaling $\left[6^{\bullet \bullet}, 10,11^{\bullet \bullet}\right]$.

In early studies, TORC1 was characterized by its sensitivity to rapamycin, while TORC2 is insensitive to rapamycin. This was later shown to be because of the ability of rapamycin (in complex with FKBP) to bind directly to TORC1 but not to TORC2. In Arabidopsis thaliana, TORC1 is insensitive to rapamycin due to the absence of a functional FKBP12 [12-14]. In T. brucei, TORC1 is insensitive to rapamycin-FKBP12 inhibition while TORC2 is sensitive. In this case, the rapamycin-FKBP12 complex can bind only to free TOR2 and not to a fully 


\begin{tabular}{|c|c|c|c|c|c|c|c|c|c|}
\hline \multicolumn{10}{|c|}{$\begin{array}{l}\text { Listed are the protein components of S. cerevisiae TORC1 and TORC2 and known homologs in other invertebrates (and mammals) in } \\
\left.\text { which TOR has been at least partly characterized. PRR5 has been suggested to be a weak homolog of BIT61 [6 } 6^{\circ}\right] . \text { TOR has also been } \\
\text { characterized in Apis mellifera and Zea mays } L \text { but no other TORC components have been studied so far in these organisms. }-=\text { no } \\
\text { homolog found or the homologs are not known to be part of the complex }\end{array}$} \\
\hline S. cerevisiae & $\begin{array}{c}\text { S. } \\
\text { pombe }\end{array}$ & $\begin{array}{c}\text { C. } \\
\text { albicans }\end{array}$ & $\begin{array}{c}D . \\
\text { discoideum }\end{array}$ & $\begin{array}{c}\text { C. } \\
\text { reinhardtii }\end{array}$ & $\begin{array}{c}T . \\
\text { brucei }\end{array}$ & $\begin{array}{c}\text { A. } \\
\text { thaliana }\end{array}$ & $\begin{array}{l}\text { C. } \\
\text { elegans }\end{array}$ & $\begin{array}{c}D . \\
\text { melanogaster }\end{array}$ & Mammals \\
\hline \multicolumn{10}{|l|}{ TORC1 } \\
\hline TOR1 or TOR2 & $\begin{array}{l}\text { TOR1 or } \\
\text { TOR2 }\end{array}$ & TOR1 & TOR & TOR & TOR1 & TOR & TOR & TOR & mTOR \\
\hline KOG1 & MIP1 & - & - & - & RAPTOR & $\begin{array}{l}\text { Raptor1A } \\
\text { Raptor1B }\end{array}$ & DAF15 & RAPTOR & RAPTOR \\
\hline LST8 & WAT1 & - & - & LST8 & - & - & LST8 & LST8 & mLST8 \\
\hline TCO89 & TCO89 & TCO89 & - & - & - & - & - & - & \\
\hline - & TOC1 & - & - & - & - & - & - & - & \\
\hline- & - & - & - & - & - & - & - & - & PRAS40 \\
\hline \multicolumn{10}{|l|}{ TORC2 } \\
\hline TOR2 & $\begin{array}{l}\text { TOR1 or } \\
\text { TOR2 }\end{array}$ & TOR1 & TOR & TOR & TOR2 & TOR & TOR & TOR & mTOR \\
\hline AVO1 & SIN1 & - & PiaA & - & - & - & SINH1 & SIN1 & mSIN1 \\
\hline AVO2 & - & - & - & - & - & - & - & - & \\
\hline AVO3 & STE20 & - & RIP3 & - & AVO3 & - & RICT1 & RICTOR & RICTOR \\
\hline LST8 & WAT1 & - & LST8 & LST8 & - & - & LST8 & LST8 & mLST8 \\
\hline BIT61 & BIT61 & - & - & - & - & - & - & - & PRR5/PRR5L \\
\hline
\end{tabular}

assembled TORC2 complex, suggesting that rapamycin in T. brucei affects the assembly and not the stability of the complex $\left[9^{\circ}\right]$, as shown previously in mammalian cells [15]. Finally, in fission yeast both TORC1 and TORC2 seem to be sensitive to rapamycin, although not under all conditions or for all readouts $\left[5,6^{\bullet \bullet}, 16-18\right]$.

While the composition of TORC1 and TORC2 are extensively described, their three-dimensional structures are far less well known. In 2007, the Llorca group reconstructed a low-resolution 3D structure of yeast TOR1 and the TOR1-KOG1 complex, by electron microscopy. Although the structure is only partial, it suggests that the RNC (Raptor N-terminal Conserved) domain of KOG1, which is known to interact with TORC1 substrates, is in close proximity to the kinase domain of TOR1 [19]. This finding begins to provide an explanation of how KOG1 might present substrates to the catalytic region of TOR1 within TORC1. Currently there is no structure of TORC2 and its resolution would be of great interest to better understand the regulation of TORC2 activity.

The intracellular localization of TOR and the TORGs has been debated for several years. In $S$. cerevisiae, early studies localized TORC1 at the plasma membrane, endosomes, the vacuolar membrane, or in the nucleus [20,21]. During the last couple of years, the issue of TOR localization was revisited. In $S$. cerevisiae, both TORC1 and TORC2 were shown to co-fractionate biochemically with endosomal membranes [22]. However, visualization of GFP-tagged versions of TOR1, TOR2 and their partners showed that TORC1 is localized to the vacuolar mem- brane $\left[23,24^{\bullet}, 25^{\bullet \bullet}, 26^{\bullet}\right]$ while TORC2 is localized to the cytoplasm and discrete sites at the plasma membrane $\left[24^{\bullet}, 26^{\circ}\right]$. In $S$. pombe, GFP-tagged SpTOR2 exhibits uneven cytoplasmic localization which changes to undefined membranous perinuclear structures upon entry into G0 $\left[6^{\bullet \bullet}\right]$. In the green algae $C$. reinhardtii, the TOR and LST8 homologs were found by biochemical methods to be associated with ER membranes, and by indirect immunofluorescence to be localized at 'dot' structures near the plasma membrane and the basal body which is enriched in ER membranes [8]. In trypanosomes, TORC1 localizes mainly to the nucleus, a localization already observed in yeast and mammalian cells, and TORC2 was found to be associated with the ER and mitochondria $\left[9^{\bullet}, 21,27,28\right]$. Altogether, the existence of TORC1 and TORC2 at diverse subcellular locations provides a molecular basis for the broad and distinct functions of the two TORCs.

\section{TORC1 signaling and cell growth Upstream regulators of TORC1}

TORC1 is a key hub mediating temporal control of cell growth, by sensing a variety of extra and intracellular growth cues such as nutrients, especially nitrogen sources and amino acids availability, growth hormones (insulin/ IGF), cellular energy (ATP), oxygen levels, and noxious stress [4]. In multicellular organisms, the best characterized upstream signaling cascade regulating TORC1 is the insulin-PI3K-TSC-Rheb pathway [4]. In this pathway, insulin-PI3K signaling inhibits the GTPase activating protein (GAP) activity of the TSC complex (a heterodimeric GAP consisting of the two proteins TSC1 and TSC2), allowing the small GTPase Rheb to activate TORC1. While TSC-Rheb-TORC1 signaling is not 
found in S. cerevisiae (no TSC homologs), this pathway is present in the fission yeast $S$. pombe and in the filamentous fungal pathogen $C$. albicans $\left[5,6^{\bullet \bullet}, 16,29-32\right]$, indicating that the pathway appeared early in evolution. However, TSC1/2 is absent or not characterized in several organisms where TOR has been identified, including plants, worms, slime mold, and T. brucei (some of which evolved later than yeasts).

The translationally controlled tumor protein (TCTP), recently identified in Drosophila as a Guanine Exchange Factor (GEF) for Rheb, positively regulates Rheb and downstream TORC1 signaling [33]. While the role of TCTP in Rheb-mTORC1 signaling is not conserved in mammals, RNAi-mediated knockdown of TCTP in $A$. thaliana leads to growth phenotypes similar to loss of AtTOR, suggesting that TCTP is involved in TOR signaling in plants [34-36]. Furthermore, TORC1 in Drosophila seems to be regulated indirectly through proteasomal degradation [37]. Indeed, TSC2 can be degraded in an ubiquitin-dependent and E3 ubiquitin ligase complex (FBW5-DDB1-CUL4 complex)-dependent manner. This underscores the complexity of TORC1 regulation by the TSC-Rheb axis.

Amino acids and nitrogen sources are well known regulators of TORC1 in several organisms; however, the molecular mechanism that triggers TORC1 activation specifically by amino acids was unknown until recently. Two new activators of TORC1 in response to amino acids were identified in D. melanogaster. The MAP kinase homolog MAP4K3 activates TORC1 leading to the phosphorylation of S6K and 4EBP in response to amino acids but not to insulin [38 $8^{\circ}$. A dimeric complex of the two small GTPases RagA and RagC also activates TORC1 specifically in response to amino acids, independently of the TSC complex and downstream or in parallel to Rheb $\left[39^{\bullet \bullet}, 40\right]$. The Rag complex affects cell growth and organ size in a nutrient-dependent manner and inhibits autophagy and starvation induced cell death $\left[39^{\bullet \bullet}, 40\right]$. The underlying molecular mechanism is likely conserved in $S$. cerevisiae because the vacuolar proteins GTR1 and GTR2 (homologs of RagA and RagC) are involved in microautophagy and have been proposed to mediate the amino acid signal to TORC1 $[20,41]$. This notion is supported by the finding that GTR1 and GTR2 are required to inhibit nitrogen catabolite-repressed (NCR) genes, which are well known target genes of TORC1 signaling, and that GTR deletion causes rapamycin hypersensitivity, an indication of a defect in TORC1 signaling $[42,43]$.

In $S$. cerevisiae, membrane trafficking to the vacuole appears to be important to trigger the amino acid signal to TORC1. Indeed, several recent studies have shown that TORC1 co-fractionates and genetically interacts with several proteins involved in protein sorting to the vacuole, including the class $\mathrm{C}$ and class D VPS (Vacuolar
Protein Sorting) proteins [22,44,45]. Mutants defective in these VPS proteins exhibit rapamycin hypersensitivity, reduced intracellular amino acid pools, and constitutive GLN3 nuclear accumulation [44,45] - all phenotypes suggesting a defect in TORC1 signaling. These findings, together with the localization of TORC1 at the vacuolar membrane and at the endosome, suggest a model in which intracellular protein trafficking (from Golgi to endosomes and from endosomes to vacuole) controls the level of intracellular amino acids which in turn affect TORC1 activity at the vacuolar membrane and at endosomes.

\section{Targets and processes downstream of TORC1}

TORC1 mediates temporal control of cell growth in response to nutrients and, in metazoans, growth factors. The growth-related processes controlled by TORC1 include transcription, protein synthesis, ribosome biogenesis, autophagy, stress responses, and nutrient transport. Despite the large number on TORC1-regulated processes, only a few direct TORC1 substrates have been identified. In metazoans, the best characterized substrates for TORC1 are the translational inhibitor 4EBP and the S6 kinase S6K [4]. In Drosophila, the dephosphorylation of S6K upon rapamycin treatment or amino acid withdrawal depends on the activity of the type $2 \mathrm{~A}$ phosphatase PP2A, linking two well known TORC1 functions - activation of kinases and inhibition of phosphatases [46]. TORC1-S6K signaling seems to be conserved in yeast. In S. cerevisiae, the AGC kinase and S6K homolog SCH9 is directly phosphorylated and activated by TORC1 $\left[25^{\bullet \bullet}\right]$. SCH9 is required for TORC1-dependent regulation of ribosome biogenesis, control of translation initiation via phosphorylation of the S6 ortho$\log$, activation of RNA polymerase III through direct phosphorylation and inhibition of the transcriptional repressor MAF1, and inhibition of entry into G0 phase via direct phosphorylation of the kinase RIM15 $\left[25^{\bullet \bullet}, 47,48\right]$. Contrary to the known inhibitory effect of noxious stresses on TORC1-SCH9 $\left[25^{\bullet \bullet}, 47,49\right]$, osmotic stress seems to promote the binding of SCH9 to the promoter of osmostress-responsive genes where it acts as a transcriptional activator [50]. This finding together with genome-wide expression profiling suggests that SCH9 also has TORC1-independent functions in yeast [50,51].

One of the major roles of TORC1 in the promotion of cell growth is the positive regulation of ribosome biogenesis and function [4,52]. In yeast, TORC1 regulates ribosomal protein $(\mathrm{RP})$ gene and ribosomal biogenesis ( $\mathrm{RiBi}$ ) gene expression through SCH9 [25 $5^{\bullet}$ ] and through the control of at least two transcription factors, FHL1 and SFP1 [4,52]. Interestingly, it was recently shown in yeast that TORC1 binds and phosphorylates SFP1 directly, in a rapamycin sensitive manner, and that this phosphorylation by TORC1 is essential to maintain SFP1 in the 
nucleus where it can activate RP and RiBi gene expression $\left[53^{\circ}\right]$. The authors of this study propose that SFP1 is a functional homolog of the proto-oncogene c-MYG that regulates $\mathrm{RP}$ and Ribi gene transcription in mammals $\left[52,53^{\bullet}, 54\right]$. This notion was recently strengthened by the finding that MYG in flies is downstream of TORC1 in activating cell growth, by promoting Ribi gene expression in response to nutrients $\left[55^{\circ}, 56^{\circ}\right]$. MYC binds and activates several Ribi genes in a TORC1-dependent manner [56 $6^{\circ}$. Furthermore, an overgrowth phenotype due to TSC1 knockdown in flies is completely abolished in the absence of MYC, while the effect of MYC overexpression in muscle cell endoreplication is abolished by TSC overexpression $\left[55^{\bullet}, 56^{\bullet}\right]$. However, MYC is regulated not only via the TOR signaling pathway but

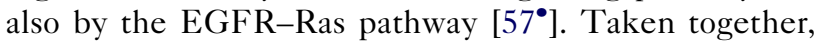
these data support a model in which MYC in flies is a downstream effector of TOR signaling in regulating Ribi gene expression and cell growth in response to nutrients.

TORC1 also regulates other factors involved in RP gene expression or ribosome biogenesis. In yeast, TORC1 regulates the subcellular localization of the $40 \mathrm{~S}$ ribosome biogenesis factor DIM2, and the function of HMO1 in the regulation of rRNA and RP genes expression [58,59]. In Drosophila, TORC1 controls the RNA pol I dependent transcription factor TIF-IA in the regulation of rRNA synthesis, similarly to what was observed for the regulation of TIF-IA homologs in yeast and mammals [60-62].

In response to nutrients, TORC1 inhibits autophagy, a process of bulk degradation of protein and organelles by the vacuole/lysosome [63]. Autophagy is mediated by a number of conserved ATG proteins each of which acts at a specific step in this process. Activation of the protein kinase ATG1 is a critical step in the induction and formation of the preautophagosomal structure (PAS). In yeast, TORC1 inactivation upon starvation leads to the dephosphorylation of ATG13 (see Figure 1). Dephosphorylated ATG13 then forms a complex with ATG1 and ATG17 in which the ATG1 kinase is activated [64]. At the signaling level, it appears that both SCH9 and the type 2A phosphatases, two well known TORC1 targets, are also implicated in the regulation of autophagy $[65,66]$. However, it should be noted that SCH9 cooperates with PKA to inhibit autophagy and that this effect seems to be partially independent of TORC1 activity [65]. The ATG1-ATG13 complex is conserved in Drosophila as a nutrient-sensitive regulator of autophagy. In flies, ATG1 interacts with TOR, and the ATG1-ATG13 complex is phosphorylated in a TOR-dependent and ATG1-dependent manner (see Figure 1). However, contrary to what is observed in yeast, ATG13 in flies is hyperphosphorylated even during starvation and remains associated with ATG1 during feeding conditions [67]. Interestingly, in flies (and mammalian cells), overexpression of ATG1 inhibits TORG1 signaling while ATG1 disruption increases TORC1 activity as measured by variation in TOR-dependent $56 \mathrm{~K}$ phosphorylation $\left[68^{\bullet}, 69^{\bullet}\right]$. Furthermore, overexpression of ATG1 or depletion of ATG13 significantly affects the cellular localization of TOR in flies [67]. These results suggest a model whereby ATG1-ATG13, by antagonizing TORC1, is involved in a self-reinforcing feedback loop $\left[67,68^{\bullet}, 69^{\bullet}\right]$ (see Figure 1).

A second target of TORC1 during autophagy in Drosophila might be the class III PI3K VPS34 (see Figure 1). VPS34 is activated by TORC1 and ATG1 and is required for TORC1-dependent and ATG1-dependent PAS formation during starvation [70]. These findings suggest that VPS34 is a downstream target of nutrient-TORC1ATG1 signaling, contrary to what has been observed in mammals where VPS34 seems to be upstream of mTORC1 [71]. The regulation of VPS34 by TORC1, as seen in flies, might be conserved in $S$. cerevisiae. A VPS34 deletion in yeast is synthetic lethal with a TOR1 deletion, and a VPS34 deletion impairs GLN3-dependent gene expression (a well known TORC1 readout) in response to poor nitrogen source [44,45]. Finally, as discussed below, autophagy has emerged as an important process in the regulation of longevity by TORC1 in flies, worms, and yeast.

\section{TORC1 and aging}

Over the last five years, TORC1 has been shown to promote aging in invertebrates. Similar to the well known negative effect of dietary restriction (DR) on aging, inactivation of TORC1 leads to an increase in lifespan in C. elegans, D. melanogaster, and S. cerevisiae [72]. Furthermore, in yeast and worms, DR is not able to further increase lifespan when TORC1 is inactive, suggesting that DR and TORC1 are in the same pathway regulating aging [72].

During the last two years, important advances were made in understanding how TORC1 regulates lifespan. Several independent studies in worms and yeast have shown that translation capacity of the ribosomal pool, which is well known to be positively regulated by TORC1, is a determinant of longevity. In $C$. elegans, knockdown of genes encoding ribosomal proteins (RPs) or translation initiation factors, such as the S6K homolog RSKS-1 or the eIF4E homolog IFE-2, significantly increases lifespan $\left[73,74^{\bullet}, 75^{\bullet}, 76^{\bullet \bullet}\right]$. Similarly, in $S$. cerevisiae, deletion of nonessential RP genes, especially those encoding the $60 \mathrm{~S}$ ribosome subunit, also extends lifespan [77,78 $\left.{ }^{\bullet}\right]$. Contrary to what is observed in C. elegans, DR is unable to extend further the long lifespan phenotype of RP gene mutants in yeast, similar to a tor 1 mutant $\left[74^{\bullet}, 76^{\bullet \bullet}, 78^{\bullet \bullet}\right]$. This suggests that translation is an important determinant of longevity downstream of DR/TORC1 signaling. Interestingly, in $S$. cerevisiae, the nutrient-controlled transcription factor GCN4 is required for full lifespan extension in 


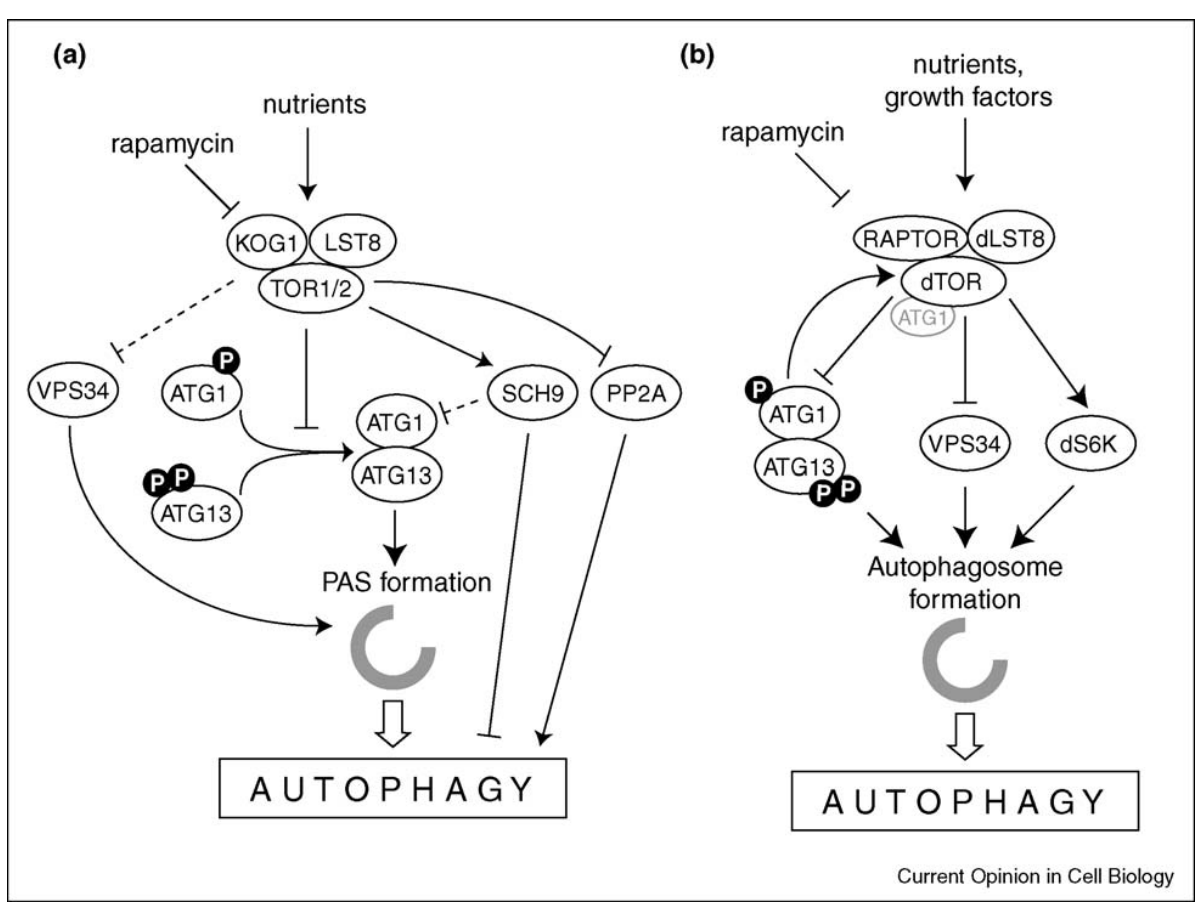

Regulation of autophagy by TORC1 in (a) S. cerevisiae and (b) D. melanogaster. In response to nutrient availability, TOR complex 1 (TOR1/TOR2/ dTOR, KOG1/RAPTOR, and LST8/dLST8) negatively regulates autophagy in yeast and flies. (a) In S. cerevisiae, TORC1 inhibits autophagy by promoting hyperphosphorylation of the kinase ATG1 and ATG13. Upon TORC1 inhibition (nutrient limitation or rapamycin treatment) ATG1 and ATG13 are dephosphorylated and associated. This leads to the formation of the preautophagosomal structure (PAS), an essential early step in autophagy. The protein kinase SCH9, a direct target of TORC1, and the TORC1 controlled type 2A phosphatase (PP2A) SIT4 inhibit and promote autophagy. The class III PI3K VPS34 is likely involved in the TORC1-dependent regulation of autophagy. (b) Contrary to yeast, in D. melanogaster, the ATG1-ATG13 interaction is not altered upon TORC1 inhibition. ATG1 interacts directly with dTOR and, once activated, signals back to TORC1 in a positive feedback loop reinforcing autophagy. VPS34 is required for the early step of autophagosome formation. The AGC kinase S6K is required for normal induction of autophagy.

response to either DR, to inhibition of TORC1-SCH9 signaling or to $60 \mathrm{~S}$ ribosome subunit depletion, placing GCN4 downstream of TORC1 in regulating lifespan $\left[78^{\bullet \bullet}\right]$.

Autophagy is another TORC1-dependent process that seems to be important for DR-dependent lifespan extension. Several analyses in worms have shown that DR induces autophagy, and that blocking autophagy by RNAi-mediated knockdown of $A T G$ genes blocks the extension of lifespan normally observed during DR or upon TORC1 loss of function (let63 and daf15 RNAi) $\left[79^{\bullet}, 80^{\bullet}, 81^{\bullet}\right]$. In Drosophila, mutation or knockdown of ATG genes shortens lifespan, while induction of autophagy by the overexpression of ATG8a in the brain reduces age-associated phenotypes and extends lifespan $\left[81^{\bullet}, 82^{\bullet}, 83^{\bullet}\right]$. Finally, in S. cerevisiae deletion of $A T G 1$ or $A T G 7$ shortens lifespan of post mitotic cells, known as chronological lifespan (CLS) [84]. Interestingly, in worms the forkhead transcription factor PHA4/FOXA seems to be a downstream effector of TORC1-S6K that promotes autophagy and longevity in response to DR $\left[79^{\bullet}, 85^{\bullet \bullet}, 86^{\bullet \bullet}\right]$. Taken together, these findings suggest a conserved mechanism in which TORC1 promotes aging through, at least, the inhibition of autophagy.

In budding yeast, different studies have highlighted the importance of the SCH9 branch of TORC1 signaling in longevity. TORC1 promotes aging through the inhibition of mitochondrial respiration [ $87^{\bullet \bullet}$ ] whereas deletion of $S C H 9$ leads to increased respiration and increased lifespan. These findings suggest that SCH9 is an important downstream target of TORC1 inhibiting mitochondrial activity during growth in the presence of glucose [88]. Furthermore, the protein kinase RIM15, which is directly phosphorylated and inhibited by SCH9, and the RIM15 target transcription factors MSN2/4 and GIS1 are required for the increase in lifespan upon TORC1 inhibition $\left[47,89^{\circ}, 90\right]$. The activation of MSN2/4 upon TORC1 inhibition promotes the expression of the prolongevity nicotinamidase gene $P N C 1$. This leads to the activation of the well-known longevity regulator SIR2, a 
histone deacetylase, suggesting that TORC1 promotes aging via negative regulation of the Sirtuin SIR2 in yeast [89 ${ }^{\circ}$. In both flies and worms, the PNC1 homolog was shown to be involved in regulating longevity and stress resistance [91,92]. However, at least in Drosophila, the nicotinamidase homolog does not seem to be regulated by TORC1 or DR [92].

It is striking that, in response to nutrients, TORC1 promotes aging in different organisms through a common set of downstream processes such as mitochondrial respiration, translation, autophagy, and transcription. Are these TORC1-regulated processes coordinated to regulate lifespan in response to nutrients? Recent findings seem to suggest an affirmative answer to this question. As shown in yeast, mature ribosomes are subject to selective, ubiquitin-dependent autophagy during nutrient starvation, linking autophagy, and translation capacity of the cell [93]. Furthermore, mRNA mistranslation in yeast induces PNC1 expression which in turn activates SIR2, linking translation efficiency and SIR2 function [94].

\section{TORC1 controls development}

Several studies in invertebrates have highlighted the role of TORC1 as a regulator of development. In the filamentous pathogenic fungus $C$. albicans, nitrogen limitation induces a switch from nonfilamentous growth to filamentous growth, leading to virulence. Recently, different reports have suggested that nitrogen limitation induces this filamentation and virulence through a conserved TSC2-RHEB-TORC1-GLN3-MEP2 signaling cascade [30,95-97]. In the plant Zea mays L (maize), TOR expression is induced during germination. While TOR is not expressed early in germination, it appears during germination and remains at this high level in different tissues at later developmental stages and in adult plants [98].

TOR is important for development in Drosophila. For example, insulin-TOR-S6K signaling appears to be a critical pathway controlling neuronal growth and differentiation [99,100]. Furthermore, when fly larvae are starved, there is a delay in the transition from larva to pupa. This delay in the timing of development is mediated by TOR in the prothoracic gland (PG), a gland that regulates the production of the hormone ecdysone. It has been suggested that a nutrient-dependent signal activates TOR in the PG which in turn leads to the production of ecdysone and subsequent pupariation [101]. Similarly, in the honeybee Apis mellifera, change in nutrient quality during feeding of female larvae leads to dimorphic caste development. While normal nutrition leads to the development of female larvae into sterile worker bees, feeding of female larvae with a particularly nutritious 'royal jelly' results in the development of queen bees. Inhibiting TOR in female larvae fed royal jelly, by rapamycin treatment or RNAi, prevents devel- opment of queen bees and results in bees with worker phenotypes [102]. Furthermore, queen development is associated with high TOR gene expression [102,103]. This suggests that TORC1 is a central switch in diphenic caste development in response to nutrients, and identifies nutrient-TORC1 signaling as a social determinant.

\section{TORC2 and growth control}

Current knowledge on TORC2 is lagging behind that on TORC1, mainly because of the lack of a good pharmacological tool, such as rapamycin, for TORC2 inhibition. However, since the identification of TORC2 in yeast [104,105] and mammals [106,107], significant progresses has been made toward a better understanding of TORC2. Below we focus on the function and regulation of TORC2 in invertebrates. In general, the pattern that seems to be emerging is that TORC2 in different organisms is more varied than TORC1, in function and regulation.

\section{Upstream regulators of TORC2}

While processes and targets downstream of TORC2 are known (actin cytoskeleton, lipid metabolism, etc.) the nature of the upstream regulators of TORC2 are poorly characterized. In Drosophila, like in mammals, TORC2 appears to be regulated by the insulin-PI3K pathway. However, in Dictyostelium discoideum, another pathway activates TORC2. In this organism, TORC2 is activated in response to a chemoattractant in a PIP3-independent manner. The activation of TORC2 by chemoattractant is mediated by heterotrimeric $G$ proteins and by RAS-like $\mathrm{G}$ proteins rasC and rasG $\left[108^{\bullet \bullet}\right.$. But other intermediate $G$ proteins are also suggested to be involved in this process. In yeast, while nitrogen source and stress are well known regulators of TORC1, their effect on TORC2 is unclear. In $S$. pombe, TOR1 is part of TORC2, but upon mild nitrogen limitation TOR1 seems also to be part of TORC1. TOR1 in TORC1 controls G2/M transition upon mild nitrogen limitation. TOR1 in TORC2 controls G1 arrest, activation of mating, and mitotic entry in response to heat and osmotic stress and upon complete nitrogen depletion $[109,110]$. These findings suggest that both TORC1 and TORC2 can be regulated by nitrogen at least in $S$. pombe. It is still unclear if the TSC1/2-RHEB pathway is able to regulate TORC2, in addition to TORC1, in $S$. pombe.

\section{Targets and processes downstream of TORC2}

Similar to TORC1, only few direct substrates have been identified for TORC2. The best characterized substrates of TORC2 are protein kinases of the AGC kinase family, such as YPK2 in S. cerevisiae and Akt/PKB in mammals, $D$. melanogaster and $C$. elegans [4,111]. TORC2 regulates these AGC kinases by phosphorylating a conserved serine or threonine in a C-terminal regulatory domain known as the hydrophobic motif. Over the last two years, this mechanism of regulation by TORC2 has shown to be conserved in other invertebrates. In $S$. pombe, TORC2 


\begin{tabular}{|c|c|c|c|c|c|}
\hline S. cerevisiae & S. pombe & D. discoideum & T. brucei & C. elegans & D. melanogaster \\
\hline$?$ & $\begin{array}{l}\text { Acute nitrogen } \\
\text { starvation, heat/ } \\
\text { osmotic stress } \\
\downarrow\end{array}$ & G protein signaling & ? & ? & $\begin{array}{l}\text { PI3K? } \\
\downarrow\end{array}$ \\
\hline$\underbrace{\text { TORC2 }}_{\mathrm{YPK} 2, \mathrm{SLM} 1 / 2}$ & $\underbrace{\text { TORC2 }}_{\text {GAD8 }}$ & $\underbrace{\text { TORC2 }}_{\text {PKBA, PKBR1 }}$ & $\frac{\text { TORC2 }}{\downarrow}$ & $\underbrace{\text { TORC2 }}_{\text {AKT, SGK1 }}$ & $\frac{\rfloor_{\text {AKT }}^{\text {TORC2 }}}{\downarrow}$ \\
\hline $\begin{array}{c}\text { Actin } \\
\text { polarization, } \\
\text { Ceramide } \\
\text { biosynthesis }\end{array}$ & $\begin{array}{l}\text { Stress response, } \\
\text { G1 arrest, } \\
\text { Mating, Mitotic } \\
\text { Entry, Amino } \\
\text { acid uptake }\end{array}$ & $\begin{array}{c}\text { Actin } \\
\text { polarization, } \\
\text { Cell motility, } \\
\text { Chemotaxis }\end{array}$ & $\begin{array}{l}\text { Cell growth, } \\
\text { Actin } \\
\text { polarization, } \\
\text { Cytokinesis }\end{array}$ & $\begin{array}{l}\text { Fat mass, } \\
\text { Growth, } \\
\text { Reproduction, } \\
\text { Feeding }\end{array}$ & $\begin{array}{c}\text { Actin } \\
\text { polarization, } \\
\text { Cell survival }\end{array}$ \\
\hline
\end{tabular}

TORC2 signaling in different invertebrates. The upstream regulators of TORC2 are largely unknown. In Schizosaccharomyces pombe, TORC2 is activated by different stresses, including acute nitrogen starvation, heat, and osmotic stress. In Dictyostelium discoideum, TORC2 is activated by chemoattractant through the activation of G-protein-coupled receptors (GPCR-cAR1) and G protein signaling. It is unknown if PI3K is upstream of TORC2 in Drosophila melanogaster. Known or assumed direct phosphorylation substrates of TORC2 in different organisms are shown. YPK2 in Saccharomyces cerevisiae, GAD8 in S. pombe, and SGK1 in C. elegans are all SGK1 homologs. AKT in C. elegans and D. melanogaster and PKBA and $\mathrm{PKBR} 1$ in $D$. discoideum are all AKT/PKB homologs. Also indicated are downstream processes controlled by TORC2.

phosphorylates the hydrophobic motif in the YPK2 homo$\log$ GAD8 to control mitotic initiation and G1 arrest in response to stress [112]. Similarly, in C. elegans, TORC2 phosphorylates and activates the YPK2 homolog SGK1 $\left[113^{\bullet \bullet}, 114^{\bullet \bullet}\right]$. This finding is supported by the observation that mTORC2 phosphorylates the hydrophobic motif of mammalian SGK1 in response to insulin [115 ${ }^{\bullet \bullet}$ ]. In D. melanogaster, TORC2 phosphorylates the hydrophobic motif in AKT, thereby activating AKT and mediating AKT-dependent inhibition of the forkhead transcription factor FOXO [116]. This activation of AKT is also found in $D$. discoideum, where TORC2 phosphorylates the AKT homolog PKBR1 in response to a chemoattractant.

Phosphorylation of the hydrophobic motif is not the only way that TORC2 regulates AGC kinases. TORC2 also phosphorylates another conserved AGG kinase region known as the turn motif. In D. melanogaster and in mammals, SIN1 knockout decreases phosphorylation of the turn motif in AK'T [117]. Similar to AKT, it has been shown in $S$. cerevisiae and mammals that a TORC2 deficiency reduces phosphorylation of the turn motif in
PKC [117]. This suggests conservation of a TORC2-PKC signaling pathway at least in yeast and mammals. In mammals, TORC2-dependent phosphorylation of the PKC $\alpha$ turn motif regulates PKC stability [117,118]. All together, these data support the notion that TORC2 is a master regulator of AGC kinases, including AKT, PKC, and YPK2/GAD8/SGK1 (Figure 2) [111].

TORC2 was originally identified as a regulator of actin cytoskeleton organization in yeast and mammals and was later shown to have a similar role in D. discoideum [4]. This function was recently shown to be conserved in the protozoan parasite $T$. bruce $i$ where TORC2 controls actin polarization and cytokinesis $\left[9^{\bullet}\right]$. In yeast and mammals, TORC2 regulates the actin cytoskeleton via Rho-type small GTPases [4]. This mechanism is likely conserved in D. discoideum, as the TORC2 substrate PKBR1 regulates GACQ, a RHO-GTPase activating protein $\left[108^{\bullet \bullet}\right.$. Recently, it was shown in $S$. cerevisiae that TORC2 controls RHO1 via two independent effector pathways. One is mediated by AVO1 and the other by AVO2 and SLM1/2 [119]. Furthermore, the recent finding that TORC2 colocalizes and genetically interacts 
with the endocytic pathway, which relies on the actin cytoskeleton, suggests that TORC2 may directly regulate endocytosis in $S$. cerevisiae [22].

TORC2 regulates lipid metabolism in several organisms. Sphingolipids, glycerolphospholipids, and sterols are the major lipids in eukaryotic membranes whose concentrations are tightly controlled. For a long time, there were hints suggesting the involvement of TORC2 in sphingolipid metabolism in S. cerevisiae, first genetic evidence [120] and later through the discovery of SLM1/2 as a TORC2 substrate [121-123]. More recently, the temperature sensitive avo3-30 mutation, a new TORC2 mutant allele defective in AVO3, allowed the analysis of sphingolipid content in S. cerevisiae and revealed that TORC2 mediates de novo ceramide synthesis. The avo3-30 mutant displays a strong reduction in the major yeast ceramide (phytosphingosine), because of reduced ceramide synthase activity. TORC2 controls ceramide synthase via phosphorylation of YPK2. Furthermore, previous reports suggested that calcineurin antagonizes TORC2 $[121,124]$, and it was shown recently that inhibition of calcineurin, by deletion of its regulatory subunit CNB1, restores ceramide levels in the avo3-30 mutant. The regulation of ceramide synthase is a novel function of TORC2 signaling, separate from TORC2-dependent actin polarization [125 ${ }^{\circ}$. The link between TORC2 and lipid metabolism is conserved in multicellular organisms. In $C$. elegans, mutants defective in RICTOR accumulate triacylglycerol (fat mass), and show developmental delay, small body size, smaller brood size, and abnormal feeding behavior. These observed phenotypes are due mainly to a defect in TORC2-SGK1 signaling rather than to a defect in TORC2-mediated activation of AKT or PKC. Only the fat mass phenotype of the RICTOR mutant could be attributed to a defect in AKT (and SGK1) activation. Thus, SGK1 appears to be the major downstream effector of TORC2. However, TORC2 clearly regulates more than SGK1 in C. elegans, since an $S G K 1$-active allele cannot suppress the phenotypes of a strong RICTOR mutant $\left[113^{\bullet \bullet}, 114^{\bullet \bullet}\right]$. TORC2 also regulates SGK1 in mammals [115 ${ }^{\bullet \bullet}$, but it remains to be determined whether mTORC2 controls lipid synthesis.

Finally, while TORC2 is essential in yeasts and mammals, flies lacking the TORC2 component RICTOR or SIN1 are viable and show only minor growth phenotypes despite a severe reduction in phosphorylation of the hydrophobic motif in AKT [126]. However, similar to what is observed in a mouse model for prostate cancer, TORC2 in flies becomes essential for hyperplasia caused by a high level of PI3K signaling [126,127]. This suggests that the importance of TORC2 in cell growth regulation differs depending on the organism and physiological context.

\section{Future directions}

Although research on TOR signaling in invertebrates continues to make important contributions, many questions remain unanswered. The observation that both TORC1 and TORC2 are at multiple locations in the cell suggests that there are functionally different subpopulations of each TORC. It would be of interest to assign location-specific functions to the TORCs. The different localizations of the TOR complexes, together with the many different biological processes regulated by TORC1 and TORC2, also suggest that there are many direct substrates remaining to be identified. The identification of these substrates either by targeted or by proteomic approaches, will likely be a major goal in the future. Furthermore, mechanisms that determine localization of the TORCs, possibly in response to growth conditions, would also be of interest. Another important, understudied aspect of TOR signaling is how and where TOR complexes are assembled and if this biogenesis is regulated.

While biological readouts of TORC1 are relatively well established, the exact mechanism by which nutrients act on TORC1 and the direct substrates of TORC1 are largely unknown. In Drosophila (and mammals), how do amino acids feed into the RAG proteins and MAP4K3 to regulate TORC1? What is the molecular mechanism by which signals are relayed to TORC1, particularly in $S$. cerevisiae, worms and plants where the TSC1/2-RHEB axis does not exist or is incomplete? The upstream regulators and direct substrates of TORC2 are even less well known. Thus, research on TOR signaling in invertebrates should remain an active area in the coming years.

\section{Acknowledgements}

We acknowledge support from the Swiss National Science Foundation and the Canton of Basel.

\section{References and recommended reading}

Papers of particular interest, published within the period of review, have been highlighted as:

- of special interest

•• of outstanding interest

1. Sehgal SN, Baker H, Vezina C: Rapamycin (AY-22,989), a new antifungal antibiotic. II. Fermentation, isolation and characterization. J Antibiot (Tokyo) 1975, 28:727-732.

2. Vezina C, Kudelski A, Sehgal SN: Rapamycin (AY-22,989), a new antifungal antibiotic. I. Taxonomy of the producing streptomycete and isolation of the active principle. J Antibiot (Tokyo) 1975, 28:721-726.

3. Heitman J, Movva NR, Hall MN: Targets for cell cycle arrest by the immunosuppressant rapamycin in yeast. Science 1991, 253:905-909.

4. Wullschleger S, Loewith $\mathrm{R}$, Hall $\mathrm{MN}$ : TOR signaling in growth and metabolism. Cell 2006, 124:471-484.

5. Matsuo T, Otsubo Y, Urano J, Tamanoi F, Yamamoto M: Loss of the TOR kinase Tor2 mimics nitrogen starvation and activates the sexual development pathway in fission yeast. Mol Cell Biol 2007, 27:3154-3164. 
6. Hayashi T, Hatanaka M, Nagao K, Nakaseko Y, Kanoh J,

- Kokubu A, Ebe M, Yanagida M: Rapamycin sensitivity of the Schizosaccharomyces pombe tor2 mutant and organization of two highly phosphorylated TOR complexes by specific and common subunits. Genes Cells 2007, 12:1357-1370.

Although several groups described the TORCs in S. pombe, this study is the most comprehensive characterization of the TOR complexes in $S$. pombe, identifying all the subunits of TORC1 and TORC2 and demon-

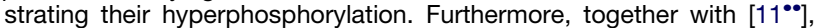
Hayashi et al. identified TEL2 as a general regulator of PIKKs.

7. Zheng C, Yan Z, Liu W, Jiang L: Identification and characterization of a functional Candida albicans homolog of the Saccharomyces cerevisiae TCO89 gene. FEMS Yeast Res 2007, 7:558-568.

8. Diaz-Troya S, Florencio FJ, Crespo JL: Target of rapamycin and LST8 proteins associate with membranes from the endoplasmic reticulum in the unicellular green alga Chlamydomonas reinhardtii. Eukaryot Cell 2008, 7:212-222.

9. Barquilla A, Crespo JL, Navarro M: Rapamycin inhibits

- $\quad$ trypanosome cell growth by preventing TOR complex 2 formation. Proc Natl Acad Sci U S A 2008, 105:14579-14584.

This study first identified TORC1 and TORC2 in Trypanosoma brucei, showing their function, localization, and their importance in growth control.

10. Kanoh J, Yanagida M: Tel2: a common partner of PIK-related kinases and a link between DNA checkpoint and nutritional response? Genes Cells 2007, 12:1301-1304.

11. Takai $\mathrm{H}$, Wang $\mathrm{RC}$, Takai KK, Yang $\mathrm{H}$, de Lange $\mathrm{T}$ : Tel2 regulates

-. the stability of PI3K-related protein kinases. Cell 2007 , 131:1248-1259.

This paper, together with Ref. [6 $6^{\bullet \bullet}$ ], identified TEL2 as a general regulator of PIKK. Takai et al. showed that Tel2 binds and stabilizes mTOR. Tel2 regulates the stability of all mammalian PIKK family members.

12. Mahfouz MM, Kim S, Delauney AJ, Verma DP: Arabidopsis TARGET OF RAPAMYCIN interacts with RAPTOR, which regulates the activity of $\mathbf{S 6}$ kinase in response to osmotic stress signals. Plant Cell 2006, 18:477-490.

13. Menand B, Desnos T, Nussaume L, Berger F, Bouchez D, Meyer C, Robaglia C: Expression and disruption of the Arabidopsis TOR (target of rapamycin) gene. Proc Natl Acad Sci U S A 2002 99:6422-6427.

14. Sormani R, Yao L, Menand B, Ennar N, Lecampion C, Meyer C, Robaglia C: Saccharomyces cerevisiae FKBP12 binds Arabidopsis thaliana TOR and its expression in plants leads to rapamycin susceptibility. BMC Plant Biol 2007, 7:26.

15. Sarbassov DD, Ali SM, Sengupta S, Sheen JH, Hsu PP, Bagley AF, Markhard AL, Sabatini DM: Prolonged rapamycin treatment inhibits mTORC2 assembly and Akt/PKB. Mol Cell 2006, 22:159-168.

16. Weisman R, Roitburg I, Schonbrun M, Harari R, Kupiec M: Opposite effects of tor1 and tor2 on nitrogen starvation responses in fission yeast. Genetics 2007, 175:1153-1162.

17. Otsubo $\mathrm{Y}$, Yamamato M: TOR signaling in fission yeast. Crit Rev Biochem Mol Biol 2008, 43:277-283.

18. Weisman R: The fission yeast TOR proteins and the rapamycin response: an unexpected tale. Curr Top Microbiol Immunol 2004, 279:85-95.

19. Adami A, Garcia-Alvarez B, Arias-Palomo E, Barford D, Llorca O: Structure of TOR and its complex with KOG1. Mol Cell 2007, 27:509-516.

20. Rohde JR, Bastidas R, Puria R, Cardenas ME: Nutritional control via Tor signaling in Saccharomyces cerevisiae. Curr Opin Microbiol 2008, 11:153-160.

21. Tsang CK, Zheng XF: TOR-in(g) the nucleus. Cell Cycle 2007, 6:25-29.

22. Aronova S, Wedaman K, Anderson S, Yates J 3rd, Powers T: Probing the membrane environment of the TOR kinases reveals functional interactions between TORC1, actin, and membrane trafficking in Saccharomyces cerevisiae. Mol Biol Cell 2007, 18:2779-2794.
23. Araki T, Uesono $\mathrm{Y}$, Oguchi T, Toh EA: LAS24/KOG1, a component of the TOR complex 1 (TORC1), is needed for resistance to local anesthetic tetracaine and normal distribution of actin cytoskeleton in yeast. Genes Genet Syst 2005, 80:325-343.

24. Berchtold D, Walther TC: TORC2 plasma membrane localization - is essential for cell viability and restricted to a distinct domain. Mol Biol Cell 2009, 20:1565-1575.

This study, together with Ref. [26"], examined the localization of the TOR complexes in live yeast cells. Berchtold et al. showed the localization of TORC2 subunits at the plasma membrane and showed that this localization is essential for TORC2 activity.

25. Urban J, Soulard A, Huber A, Lippman S, Mukhopadhyay D,

-. Deloche O, Wanke V, Anrather D, Ammerer G, Riezman H et al.: Sch9 is a major target of TORC1 in Saccharomyces cerevisiae. $\mathrm{Mol}$ Cell 2007, 26:663-674.

The authors identified SCH9 as a direct substrate of TORC1 in S. cerevisiae and proposed that SCH9 is the functional homolog of S6K.

26. Sturgill TW, Cohen A, Diefenbacher M, Trautwein M, Martin DE,

- Hall MN: TOR1 and TOR2 have distinct locations in live cells. Eukaryot Cell 2008, 7:1819-1830.

This study first examined the localization of the TOR complexes in live cells. Sturgill et al. showed that TOR2 is localized at the plasma membrane while TOR1 is localized at the vacuolar membrane.

27. Zhang X, Shu L, Hosoi H, Murti KG, Houghton PJ: Predominant nuclear localization of mammalian target of rapamycin in normal and malignant cells in culture. J Biol Chem 2002 277:28127-28134.

28. Li H, Tsang CK, Watkins M, Bertram PG, Zheng XF: Nutrient regulates Tor1 nuclear localization and association with rDNA promoter. Nature 2006, 442:1058-1061.

29. Aspuria PJ, Sato T, Tamanoi F: The TSC/Rheb/TOR signaling pathway in fission yeast and mammalian cells: temperature sensitive and constitutive active mutants of TOR. Cell Cycle 2007, 6:1692-1695.

30. Tsao CC, Chen YT, Lan CY: A small G protein Rhb1 and a GTPase-activating protein Tsc2 involved in nitrogen starvation-induced morphogenesis and cell wall integrity of Candida albicans. Fungal Genet Biol 2009, 46:126-136.

31. Urano J, Sato $T$, Matsuo $T$, Otsubo $Y$, Yamamoto M, Tamanoi F: Point mutations in TOR confer Rheb-independent growth in fission yeast and nutrient-independent mammalian TOR signaling in mammalian cells. Proc Natl Acad Sci U S A 2007, 104:3514-3519.

32. Uritani M, Hidaka H, Hotta Y, Ueno M, Ushimaru T, Toda T: Fission yeast Tor2 links nitrogen signals to cell proliferation and acts downstream of the Rheb GTPase. Genes Cells 2006, 11:1367-1379.

33. Hsu YC, Chern JJ, Cai Y, Liu M, Choi KW: Drosophila TCTP is essential for growth and proliferation through regulation of dRheb GTPase. Nature 2007, 445:785-788.

34. Berkowitz O, Jost R, Pollmann S, Masle J: Characterization of TCTP, the translationally controlled tumor protein, from Arabidopsis thaliana. Plant Cell 2008, 20:3430-3447.

35. Rehmann $\mathrm{H}$, Bruning M, Berghaus $\mathrm{C}$, Schwarten $\mathrm{M}$, Kohler $\mathrm{K}$ Stocker H, Stoll R, Zwartkruis FJ, Wittinghofer A: Biochemical characterisation of TCTP questions its function as a guanine nucleotide exchange factor for Rheb. FEBS Lett 2008 , 582:3005-3010.

36. Wang $\mathrm{X}$, Fonseca $\mathrm{BD}$, Tang $\mathrm{H}$, Liu $\mathrm{R}$, Elia A, Clemens $\mathrm{MJ}$, Bommer UA, Proud CG: Re-evaluating the roles of proposed modulators of mammalian target of rapamycin complex 1 (mTORC1) signaling. J Biol Chem 2008, 283:30482-30492.

37. Hu J, Zacharek S, He YJ, Lee H, Shumway S, Duronio RJ, Xiong Y: WD40 protein FBW5 promotes ubiquitination of tumor suppressor TSC2 by DDB1-CUL4-ROC1 ligase. Genes Dev 2008, 22:866-871.

38. Findlay GM, Yan L, Procter J, Mieulet V, Lamb RF: A MAP4 kinase

- related to $S t e 20$ is a nutrient-sensitive regulator of $\mathrm{mTOR}$ signalling. Biochem J 2007, 403:13-20. 
In this paper, the authors show that the MAP kinase MAP4K3 specifically activates TORC1 in response to amino acids in Drosophila melanogaster and mammals.

39. Kim E, Goraksha-Hicks P, Li L, Neufeld TP, Guan KL: Regulation - of TORC1 by Rag GTPases in nutrient response. Nat Cell Biol 2008, 10:935-945.

This study showed that in both flies and mammals the Rag GTPases activate mTORC1 in response to amino acids but not insulin.

40. Kim E, Guan KL: RAG GTPases in nutrient-mediated TOR signaling pathway. Cell Cycle 2009, 8:1014-1018.

41. Dubouloz F, Deloche O, Wanke V, Cameroni E, De Virgilio C: The TOR and EGO protein complexes orchestrate microautophagy in yeast. Mol Cell 2005, 19:15-26.

42. Sekiguchi T, Hayashi N, Wang Y, Kobayashi H: Genetic evidence that Ras-like GTPases, Gtr1p, and Gtr2p, are involved in epigenetic control of gene expression in Saccharomyces cerevisiae. Biochem Biophys Res Commun 2008, 368:748-754.

43. Wang $Y$, Kurihara $Y$, Sato $T$, Toh $H$, Kobayashi $H$, Sekiguchi $T$ : Gtr1p differentially associates with Gtr2p and Ego1p. Gene 2009, 437:32-38.

44. Zurita-Martinez SA, Puria R, Pan X, Boeke JD, Cardenas ME: Efficient Tor signaling requires a functional class $C$ Vps protein complex in Saccharomyces cerevisiae. Genetics 2007, 176:2139-2150.

45. Puria R, Zurita-Martinez SA, Cardenas ME: Nuclear translocation of GIn3 in response to nutrient signals requires Golgi-toendosome trafficking in Saccharomyces cerevisiae. Proc Natl Acad Sci U S A 2008, 105:7194-7199.

46. Bielinski VA, Mumby MC: Functional analysis of the PP2A subfamily of protein phosphatases in regulating Drosophila $\mathrm{S} 6$ kinase. Exp Cell Res 2007, 313:3117-3126.

47. Wanke V, Cameroni E, Uotila A, Piccolis M, Urban J, Loewith R, De Virgilio C: Caffeine extends yeast lifespan by targeting TORC1. Mol Microbiol 2008, 69:277-285.

48. Lee J, Moir RD, Willis IM: Regulation of RNA polymerase III transcription involves SCH9-dependent and SCH9independent branches of the Target of Rapamycin (TOR) pathway. J Biol Chem 2009, 284:12604-12608.

49. Hosiner $D$, Lempiainen $H$, Reiter $W$, Urban J, Loewith $R$, Ammerer G, Schweyen R, Shore D, Schuller C: Arsenic toxicity to Saccharomyces cerevisiae is a consequence of inhibition of the TORC1 kinase combined with a chronic stress response. Mol Biol Cell 2009, 20:1048-1057.

50. Pascual-Ahuir A, Proft M: The Sch9 kinase is a chromatinassociated transcriptional activator of osmostressresponsive genes. EMBO J 2007, 26:3098-3108.

51. Smets B, De Snijder P, Engelen K, Joossens E, Ghillebert R, Thevissen K, Marchal K, Winderickx J: Genome-wide expression analysis reveals TORC1-dependent and -independent functions of Sch9. FEMS Yeast Res 2008, 8:1276-1288.

52. Cook M, Tyers M: Size control goes global. Curr Opin Biotechnol 2007, 18:341-350.

53. Lempiainen H, Uotila A, Urban J, Dohnal I, Ammerer G, Loewith R,

- Shore D: Sfp1 interaction with TORC1 and Mrs6 reveals feedback regulation on TOR signaling. Mol Cell 2009, 33:704-716.

This study showed that the transcription factor SFP1, a regulator of RP and Ribi genes, is a substrate of TORC1 in Saccharomyces cerevisiae.

54. Dang CV, O'Donnell KA, Zeller KI, Nguyen T, Osthus RC, Li F: The c-Myc target gene network. Semin Cancer Biol 2006 16:253-264.

55. Demontis F, Perrimon N: Integration of insulin receptor/Foxo - $\quad$ signaling and dMyc activity during muscle growth regulates body size in Drosophila. Development 2009, 136:983-993.

Together with $\left[56^{\circ}, 57^{\circ}\right]$, this paper showed that the MYC transcription factor is a downstream effector of TORC1 signaling in Drosophila melanogaster. Demnontis and Perrimon demonstrate that MYC is an important effector of TSC/TOR in muscle endoreplication and growth.
56. Teleman AA, Hietakangas V, Sayadian AC, Cohen SM: Nutritional - control of protein biosynthetic capacity by insulin via Myc in Drosophila. Cell Metab 2008, 7:21-32.

Together with $\left[55^{\circ}, 57^{\circ}\right]$, this paper showed that the MYC transcription factor is a downstream effector of TORC1 signaling in Drosophila melanogaster. Teleman et al. provide genetic evidence that MYC has a key role in mediating the effects of TOR and FOXO on growth and metabolism.

57. Read RD, Cavenee WK, Furnari FB, Thomas JB: A drosophila

- model for EGFR-Ras and PI3K-dependent human glioma. PLoS Genet 2009, 5:e1000374.

Together with $\left[55^{\circ}, 56^{\circ}\right]$, this paper shows that the MYC transcription factor is a downstream effector of TORC1 signaling in Drosophila melanogaster. Read et al. found that Myc is a key rate-limiting gene required for glial neoplasia downstream of both PI3K-TOR and EGFR-RAS signaling pathways.

58. Vanrobays E, Leplus A, Osheim YN, Beyer AL, Wacheul L, Lafontaine DL: TOR regulates the subcellular distribution of DIM2, a KH domain protein required for cotranscriptional ribosome assembly and pre-40S ribosome export. Rna 2008 , 14:2061-2073.

59. Berger AB, Decourty L, Badis G, Nehrbass U, Jacquier A, Gadal O: Hmo1 is required for TOR-dependent regulation of ribosomal protein gene transcription. Mol Cell Biol 2007, 27:8015-8026.

60. Mayer C, Zhao J, Yuan X, Grummt I: mTOR-dependent activation of the transcription factor TIF-IA links rRNA synthesis to nutrient availability. Genes Dev 2004, 18:423-434.

61. Claypool JA, French SL, Johzuka K, Eliason K, Vu L, Dodd JA, Beyer AL, Nomura M: Tor pathway regulates Rrn3p-dependent recruitment of yeast RNA polymerase I to the promoter but does not participate in alteration of the number of active genes. Mol Biol Cell 2004, 15:946-956.

62. Grewal SS, Evans JR, Edgar BA: Drosophila TIF-IA is required for ribosome synthesis and cell growth and is regulated by the TOR pathway. J Cell Biol 2007, 179:1105-1113.

63. Diaz-Troya S, Perez-Perez ME, Florencio FJ, Crespo JL: The role of TOR in autophagy regulation from yeast to plants and mammals. Autophagy 2008, 4:851-865.

64. Kamada Y, Funakoshi T, Shintani T, Nagano K, Ohsumi M, Ohsumi Y: Tor-mediated induction of autophagy via an Apg1 protein kinase complex. J Cell Biol 2000, 150:1507-1513.

65. Yorimitsu T, Zaman S, Broach JR, Klionsky DJ: Protein kinase A and Sch9 cooperatively regulate induction of autophagy in Saccharomyces cerevisiae. Mol Biol Cell 2007, 18:4180-4189.

66. Yorimitsu T, He C, Wang K, Klionsky DJ: Tap42-associated protein phosphatase type $2 \mathrm{~A}$ negatively regulates induction of autophagy. Autophagy 2009, 5:616-624.

67. Chang YY, Neufeld TP: An Atg1/Atg13 complex with multiple roles in TOR-mediated autophagy regulation. $\mathrm{Mol}$ Biol Cell 2009, 20:2004-2014.

68. Lee SB, Kim S, Lee J, Park J, Lee G, Kim Y, Kim JM, Chung J:

- ATG1, an autophagy regulator, inhibits cell growth by negatively regulating $\mathbf{S 6}$ kinase. EMBO Rep 2007, 8:360-365.

This study showed that ATG1 inhibits TOR/S6K-dependent cell growth and development by interfering with S6K activation in flies and mammals. This indicates crosstalk between autophagy and cell growth regulation.

69. Scott RC, Juhasz G, Neufeld TP: Direct induction of autophagy

- by Atg1 inhibits cell growth and induces apoptotic cell death. Curr Biol 2007, 17:1-11.

The authors showed that ATG1 expression and activation results in negative feedback on TOR.

70. Juhasz G, Hill JH, Yan Y, Sass M, Baehrecke EH, Backer JM, Neufeld TP: The class III PI(3)K Vps34 promotes autophagy and endocytosis but not TOR signaling in Drosophila. $J$ Cell Biol 2008, 181:655-666.

71. Nobukuni T, Joaquin M, Roccio M, Dann SG, Kim SY, Gulati P Byfield MP, Backer JM, Natt F, Bos JL et al.: Amino acids mediate mTOR/raptor signaling through activation of class 3 phosphatidylinositol 30H-kinase. Proc Natl Acad Sci U S A 2005, 102:14238-14243. 
72. Bishop NA, Guarente L: Genetic links between diet and lifespan: shared mechanisms from yeast to humans. Nat Rev Genet 2007, 8:835-844.

73. Curran SP, Ruvkun G: Lifespan regulation by evolutionarily conserved genes essential for viability. PLOS Genet 2007, 3:e56.

74. Hansen M, Taubert S, Crawford D, Libina N, Lee SJ, Kenyon C:

- Lifespan extension by conditions that inhibit translation in Caenorhabditis elegans. Aging Cell 2007, 6:95-110.

Together with Refs. $\left[75^{\circ}, 76^{\circ} \cdot\right.$, this study showed that translation capacity of the ribosome is an important determinant of longevity in worms. Hansen et al. reported that reducing the levels of ribosomal proteins, ribosomal-protein S6 kinase or translation-initiation factors increases the lifespan of Caenorhabditis elegans.

75. Pan KZ, Palter JE, Rogers AN, Olsen A, Chen D, Lithgow GJ,

- Kapahi $P$ : Inhibition of mRNA translation extends lifespan in Caenorhabditis elegans. Aging Cell 2007, 6:111-119.

Together with Refs. $\left[74^{\bullet}, 76^{\circ \bullet}\right]$, this study showed that translation capacity of the ribosome is an important determinant of longevity in worms. Pan et al. have showed that inhibition of various genes in the translationinitiation complex results in lifespan extension in Caenorhabditis elegans.

76. Syntichaki $P$, Troulinaki $K$, Tavernarakis $N$ : eIF4E function in

-. somatic cells modulates ageing in Caenorhabditis elegans. Nature 2007, 445:922-926.

Together with Refs. $\left[74^{\bullet}, 75^{\circ}\right]$, this study showed that translation capacity of the ribosome is an important determinant of longevity in worms. Syntichaki et al. have showed that loss of a specific elF4E isoform (IFE-2) reduces global protein synthesis and extends lifespan in Caenorhabditis elegans.

77. Chiocchetti A, Zhou J, Zhu H, Karl T, Haubenreisser O, Rinnerthaler M, Heeren G, Oender K, Bauer J, Hintner $\mathrm{H}$ et al:: Ribosomal proteins Rpl10 and Rps 6 are potent regulators of yeast replicative life span. Exp Gerontol 2007, 42:275-286.

78. Steffen KK, MacKay VL, Kerr EO, Tsuchiya M, Hu D, Fox LA

-. Dang N, Johnston ED, Oakes JA, Tchao BN et al: Yeast life span extension by depletion of 60 s ribosomal subunits is mediated by Gcn4. Cell 2008, 133:292-302.

Steffen et al. showed that specific inhibition of 605 ribosome biogenesis is able to enhance longevity similarly to DR or TORC1 inhibition in Saccharomyces cerevisiae.

79. Hansen M, Chandra A, Mitic LL, Onken B, Driscoll M, Kenyon C: A role for autophagy in the extension of lifespan by dietary restriction in C. elegans. PLOS Genet 2008, 4:e24

Along with Refs. $\left[80^{\circ}, 81^{\circ}\right]$, this paper demonstrates that the induction of autophagy upon nutrient starvation is involved in the regulation of longevity in worms and suggests a conserved mechanism where TORC1 promotes aging through the inhibition of autophagy.

80. Jia K, Levine B: Autophagy is required for dietary restriction-

- mediated life span extension in C. elegans. Autophagy 2007 3:597-599.

Along with Refs. $\left[79^{\circ}, 81^{\circ}\right]$, this paper demonstrates that the induction of autophagy upon nutrient starvation is involved in the regulation of longevity in worms and suggests a conserved mechanism where TORC1promotes aging through the inhibition of autophagy.

81. Toth ML, Sigmond T, Borsos E, Barna J, Erdelyi P, Takacs-Vellai K,

- Orosz L, Kovacs AL, Csikos G, Sass M et al.: Longevity pathways converge on autophagy genes to regulate life span in Caenorhabditis elegans. Autophagy 2008, 4:330-338.

Along with Refs. $\left[79^{\circ}, 80^{\circ}\right]$, this paper demonstrates that the induction of autophagy upon nutrient starvation is involved in the regulation of longevity in worms and suggests a conserved mechanism where TORC1promotes aging through the inhibition of autophagy.

82. Simonsen A, Cumming RC, Brech A, Isakson P, Schubert DR,

- $\quad$ Finley KD: Promoting basal levels of autophagy in the nervous system enhances longevity and oxidant resistance in adult Drosophila. Autophagy 2008, 4:176-184.

This paper demonstrates that the induction of autophagy upon nutrient starvation is involved in the regulation of longevity in flies and suggests a conserved mechanism where TORC1 promotes aging through the inhibition of autophagy.

83. Juhasz G, Erdi B, Sass M, Neufeld TP: Atg7-dependent

- autophagy promotes neuronal health, stress tolerance, and longevity but is dispensable for metamorphosis in Drosophila. Genes Dev 2007, 21:3061-3066.
Along with Ref. $\left[8^{\circ}\right]$, this paper demonstrates that the induction of autophagy upon nutrient starvation is involved in the regulation of longevity in flies and suggests a conserved mechanism where TORC1 promotes aging through the inhibition of autophagy.

84. Alvers AL, Fishwick LK, Wood MS, Hu D, Chung HS, Dunn WA Jr, Aris JP: Autophagy and amino acid homeostasis are required for chronological longevity in Saccharomyces cerevisiae. Aging Cell 2009, 8:353-369.

85. Panowski SH, Wolff S, Aguilaniu H, Durieux J, Dillin A: PHA-4/

- Foxa mediates diet-restriction-induced longevity of $\mathbf{C}$ elegans. Nature 2007, 447:550-555.

Panowski et al. showed that Dietary Restriction induces longevity through the Foxo transcription factor PHA-4 in Caenorhabditis elegans.

86. Sheaffer KL, Updike DL, Mango SE: The Target of Rapamycin

- pathway antagonizes pha-4/FoxA to control development and aging. Curr Biol 2008, 18:1355-1364.

Sheaffer et al. showed that PHA-4 is required to extend adult lifespan in response to reduced TOR signaling in worms.

87. Bonawitz ND, Chatenay-Lapointe M, Pan Y, Shadel GS: Reduced

- $\quad$ TOR signaling extends chronological life span via increased respiration and upregulation of mitochondrial gene expression. Cell Metab 2007, 5:265-277.

The authors showed that TORC1 promotes aging primarily through the inhibition of mitochondrial respiration in Saccharomyces cerevisiae.

88. Lavoie $H$, Whiteway $M$ : Increased respiration in the sch9Delta mutant is required for increasing chronological life span but not replicative life span. Eukaryot Cell 2008, 7:1127-1135.

89. Medvedik O, Lamming DW, Kim KD, Sinclair DA: MSN2 and MSN4

- link calorie restriction and TOR to sirtuin-mediated lifespan extension in Saccharomyces cerevisiae. PLOS Biol 2007, 5:e261.

This study showed that, similarly to dietary restriction, TORC1 inhibition extends lifespan by increasing Sir2p activity through the upregulation of the nicotinamidase PNC1.

90. Wei M, Fabrizio P, Hu J, Ge H, Cheng C, Li L, Longo VD: Life span extension by calorie restriction depends on Rim15 and transcription factors downstream of Ras/PKA, Tor, and Sch9. PLoS Genet 2008, 4:e13.

91. van der Horst A, Schavemaker JM, Pellis-van Berkel W, Burgering BM: The Caenorhabditis elegans nicotinamidase PNC-1 enhances survival. Mech Ageing Dev 2007, 128:346-349.

92. Balan V, Miller GS, Kaplun L, Balan K, Chong ZZ, Li F, Kaplun A, VanBerkum MF, Arking R, Freeman DC et al.: Life span extension and neuronal cell protection by Drosophila nicotinamidase. $J$ Biol Chem 2008, 283:27810-27819.

93. Kraft C, Deplazes A, Sohrmann M, Peter M: Mature ribosomes are selectively degraded upon starvation by an autophagy pathway requiring the Ubp3p/Bre5p ubiquitin protease. Nat Cell Biol 2008, 10:602-610.

94. Silva RM, Duarte IC, Paredes JA, Lima-Costa T, Perrot M, Boucherie H, Goodfellow BJ, Gomes AC, Mateus DD, Moura GR et al.: The yeast PNC1 longevity gene is up-regulated by mRNA mistranslation. PLOS ONE 2009, 4:e5212.

95. Bastidas RJ, Heitman J, Cardenas ME: The protein kinase Tor1 regulates adhesin gene expression in Candida albicans. PLOS Pathog 2009, 5:e1000294.

96. Dabas N, Morschhauser J: Control of ammonium permease expression and filamentous growth by the GATA transcription factors GLN3 and GAT1 in Candida albicans. Eukaryot Cell 2007, 6:875-888.

97. Liao WL, Ramon AM, Fonzi WA: GLN3 encodes a global regulator of nitrogen metabolism and virulence of $C$. albicans. Fungal Genet Biol 2008, 45:514-526.

98. Agredano-Moreno LT, Reyes de la Cruz H, Martinez-Castilla LP, Sanchez de Jimenez E: Distinctive expression and functional regulation of the maize (Zea mays L.) TOR kinase ortholog. Mol Biosyst 2007, 3:794-802.

99. Bateman JM, McNeill H: Temporal control of differentiation by the insulin receptor/tor pathway in Drosophila. Cell 2004, 119:87-96. 
100. McNeill H, Craig GM, Bateman JM: Regulation of neurogenesis and epidermal growth factor receptor signaling by the insulin receptor/target of rapamycin pathway in Drosophila. Genetics 2008, 179:843-853.

101. Layalle S, Arquier N, Leopold P: The TOR pathway couples nutrition and developmental timing in Drosophila. Dev Cell 2008, 15:568-577.

102. Patel A, Fondrk MK, Kaftanoglu O, Emore C, Hunt G, Frederick K, Amdam GV: The making of a queen: TOR pathway is a key player in diphenic caste development. PLOS ONE 2007, 2:e509.

103. Barchuk AR, Cristino AS, Kucharski R, Costa LF, Simoes ZL, Maleszka R: Molecular determinants of caste differentiation in the highly eusocial honeybee Apis mellifera. BMC Dev Biol 2007, 7:70.

104. Loewith R, Jacinto E, Wullschleger S, Lorberg A, Crespo JL, Bonenfant D, Oppliger W, Jenoe P, Hall MN: Two TOR complexes, only one of which is rapamycin sensitive, have distinct roles in cell growth control. Mol Cell 2002, 10:457-468.

105. Wedaman KP, Reinke A, Anderson S, Yates J 3rd, McCaffery JM, Powers T: Tor kinases are in distinct membrane-associated protein complexes in Saccharomyces cerevisiae. Mol Biol Cell 2003, 14:1204-1220.

106. Jacinto $E$, Loewith R, Schmidt A, Lin S, Ruegg MA, Hall A, Hall MN: Mammalian TOR complex 2 controls the actin cytoskeleton and is rapamycin insensitive. Nat Cell Biol 2004, 6:1122-1128.

107. Sarbassov DD, Ali SM, Kim DH, Guertin DA, Latek RR, ErdjumentBromage $H$, Tempst $P$, Sabatini DM: Rictor, a novel binding partner of $\mathrm{mTOR}$, defines a rapamycin-insensitive and raptorindependent pathway that regulates the cytoskeleton. Curr Biol 2004, 14:1296-1302.

108. Kamimura $Y$, Xiong $Y$, Iglesias $P A$, Hoeller O, Bolourani $P$,

-. Devreotes PN: PIP3-independent activation of TorC2 and PKB at the cell's leading edge mediates chemotaxis. Curr Biol 2008, 18:1034-1043.

This study demonstrated the involvement of TORC2 in a PIP3 independent pathway in chemotaxis through its effector PKBR1 and the involvement of heterotrimeric $G$ proteins and other RAS like $G$ proteins in this process.

109. Petersen J, Nurse P: TOR signalling regulates mitotic commitment through the stress MAP kinase pathway and the Polo and Cdc2 kinases. Nat Cell Biol 2007, 9:1263-1272.

110. Hartmuth S, Petersen J: Fission yeast Tor1 functions as part of TORC1 to control mitotic entry through the stress MAPK pathway following nutrient stress. J Cell Sci 2009 , 122:1737-1746.

111. Jacinto $E$, Lorberg $A$ : TOR regulation of AGC kinases in yeast and mammals. Biochem $J$ 2008, 410:19-37.

112. Ikeda K, Morigasaki S, Tatebe H, Tamanoi F, Shiozaki K: Fission yeast TOR complex 2 activates the AGC-family Gad8 kinase essential for stress resistance and cell cycle control. Cell Cycle 2008, 7:358-364.

113. Jones KT, Greer ER, Pearce D, Ashrafi K: Rictor/TORC2

-. regulates Caenorhabditis elegans fat storage, body size, and development through sgk-1. PLoS Biol 2009, 7:e60

This study, together with Ref. [114**], identified rictor mutants in Caenorhabditis elegans, showing the involvement of TORC2 in the regulation of fat metabolism, development, body size, brood size, lifespan, and feeding behavior. These studies also identified SGK1 as a major effector of TORC2 in Caenorhabditis elegans.

114. Soukas AA, Kane EA, Carr CE, Melo JA, Ruvkun G: Rictor/TORC2

-. regulates fat metabolism, feeding, growth, and life span in Caenorhabditis elegans. Genes Dev 2009, 23:496-511.
This study, together with Ref. [113 ${ }^{\bullet \bullet}$, identified rictor mutants in Caenorhabditis elegans, showing the involvement of TORC2 in the regulation of fat metabolism, development, body size, brood size, lifespan, and feeding behavior.

115. Garcia-Martinez JM, Alessi DR: mTOR complex 2 (mTORC2)

- controls hydrophobic motif phosphorylation and activation of serum- and glucocorticoid-induced protein kinase 1 (SGK1). Biochem J 2008, 416:375-385.

This study identified SGK1 as a major effector of TORC2 in mammalian cells, and showed that TORC2 directly phosphorylate SGK1 in the hydrophobic motif and thus activates it.

116. Lee G, Chung J: Discrete functions of rictor and raptor in cell growth regulation in Drosophila. Biochem Biophys Res Commun 2007, 357:1154-1159.

117. Facchinetti V, Ouyang W, Wei H, Soto N, Lazorchak A, Gould C, Lowry C, Newton AC, Mao Y, Miao RQ et al.: The mammalian target of rapamycin complex 2 controls folding and stability of Akt and protein kinase C. EMBO J 2008, 27:1932-1943.

118. Ikenoue T, Inoki K, Yang Q, Zhou X, Guan KL: Essential function of TORC2 in PKC and Akt turn motif phosphorylation, maturation and signalling. EMBO J 2008, 27:1919-1931.

119. Ho HL, Lee HY, Liao HC, Chen MY: Involvement of Saccharomyces cerevisiae Avo3p/Tsc11p in maintaining TOR complex 2 integrity and coupling to downstream signaling. Eukaryot Cell 2008, 7:1328-1343.

120. Beeler T, Bacikova D, Gable K, Hopkins L, Johnson C, Slife H, Dunn T: The Saccharomyces cerevisiae TSC10/YBR265w gene encoding 3-ketosphinganine reductase is identified in a screen for temperature-sensitive suppressors of the $\mathrm{Ca}^{2+}$-sensitive csg2Delta mutant. J Biol Chem 1998 273:30688-30694.

121. Bultynck G, Heath VL, Majeed AP, Galan JM, HaguenauerTsapis R, Cyert MS: SIm1 and sIm2 are novel substrates of the calcineurin phosphatase required for heat stress-induced endocytosis of the yeast uracil permease. Mol Cell Biol 2006, 26:4729-4745.

122. Tabuchi M, Audhya A, Parsons AB, Boone C, Emr SD: The phosphatidylinositol 4,5-biphosphate and TORC2 binding proteins SIm1 and SIm2 function in sphingolipid regulation. Mol Cell Biol 2006, 26:5861-5875.

123. Daquinag A, Fadri M, Jung SY, Qin J, Kunz J: The yeast PH domain proteins SIm1 and SIm2 are targets of sphingolipid signaling during the response to heat stress. Mol Cell Biol 2007, 27:633-650.

124. Mulet JM, Martin DE, Loewith R, Hall MN: Mutual antagonism of target of rapamycin and calcineurin signaling. J Biol Chem 2006, 281:33000-33007.

125. Aronova S, Wedaman K, Aronov PA, Fontes K, Ramos K,

- Hammock BD, Powers T: Regulation of ceramide biosynthesis by TOR complex 2. Cell Metab 2008, 7:148-158.

This study was the first study to show that TORC2 controls ceramide synthesis in Saccharomyces cerevisiae on the level of ceramide synthase. This effect was shown to be mediated by YPK2 and antagonized by calcineurin and separate from the effect of TORC2 on actin.

126. Hietakangas V, Cohen SM: Re-evaluating AKT regulation: role of TOR complex 2 in tissue growth. Genes Dev 2007, 21:632-637.

127. Guertin DA, Stevens DM, Saitoh M, Kinkel S, Crosby K, Sheen JH, Mullholland DJ, Magnuson MA, Wu H, Sabatini DM: mTOR complex 2 is required for the development of prostate cancer induced by Pten loss in mice. Cancer Cell 2009, 15:148-159. 